The Journal of Innovations in Cardiac Rhythm Management, 7 (2016), 2304-2306

\title{
Remote Magnetic Navigation-guided Catheter Ablation of Atrioventricular Nodal Reentrant Tachycardia in an Adult Patient with Prior Surgical Closure of Perimembranous Ventricular Septal Defect during Infancy
}

\author{
STEFANI M. SAMPLES, MD ${ }^{1}$ and ADAM E. BERMAN, MD, FHRS ${ }^{2}$ \\ ${ }^{1}$ Department of Pediatric Cardiology, Augusta University, Children's Hospital of Georgia, Augusta, GA \\ ${ }^{2}$ Director of Cardiac Arrhythmia Ablation Services, AU Health Heart and Cardiovascular Services, Augusta, GA
}

\begin{abstract}
Ventricular septal defects (VSDs) are one of the most common types of congenital heart disease. The cardiac conduction system is intimately involved with these defects and their repair may further alter the location of that system. In patients with prior surgical repair of VSDs, special consideration should be given to carefully delineating the location of the normal conduction system during the diagnostic component of electrophysiologic testing prior to ablation delivery. Here we present a case of a 40-year-old man with a previously repaired VSD who underwent electrophysiologic testing and eventual remote magnetic navigation-guided catheter ablation of atrioventricular nodal reentry tachycardia.
\end{abstract}

KEYWORDS. atrioventricular nodal reentry tachycardia, radiofrequency ablation, remote magnetic navigation, ventricular septal defects.
ISSN 2156-3977 (print) ISSN 2156-3993 (online)

(C) 2016 Innovations in Cardiac Rhythm Management

\section{Introduction}

Radiofrequency catheter ablation (RFCA) of supraventricular tachycardias involving the septum in patients with prior surgical repair of ventricular septal defects has been poorly described. We present the initial case report of RFCA of typical atrioventricular nodal reentry tachycardia (AVNRT) using Stereotaxis remote magnetic navigation $(\mathrm{RMN})$ in an adult patient with prior surgical repair of a perimembranous ventricular septal defect (VSD).

\section{Case report}

A 40-year-old man with a history of surgical repair of a perimembranous VSD in infancy presented with complaints

Dr. Berman reports serving as a consultant for Stereotaxis, Inc. Manuscript received February 16, 2016, Final version accepted March 7, 2016.

Address correspondence to: Stefani Samples, MD, 1120 15th St BAA-8300, Augusta, GA 30912. E-mail: ssamples@gru.edu of palpitations. On one occasion, the palpitations were associated with near-syncope. His other cardiac history was remarkable for aortic valve replacement: first at age 9 years with a \#21 Bjork Shiley valve and subsequently a \#25 St. Jude Medical (St Paul, MN) valve at age 17 years.

Outpatient non-invasive electrocardiograph monitoring was unremarkable with the exception of several ventricular couplets. Transthoracic echocardiography demonstrated left ventricular (LV) ejection fraction of $50 \%$ with no evidence of residual shunt by microcavitation study. The mechanical aortic prosthesis functioned normally. On the basis of his tachycardic associated near-syncope, the decision was made to proceed with invasive electrophysiologic testing to exclude significant arrhythmia as the etiology of his near syncope.

The patient underwent EP testing with catheters positioned in the right atrium, coronary sinus, right ventricular apex, and His region. Programmed ventricular stimulation was performed with up to three extra-stimuli at two differing 


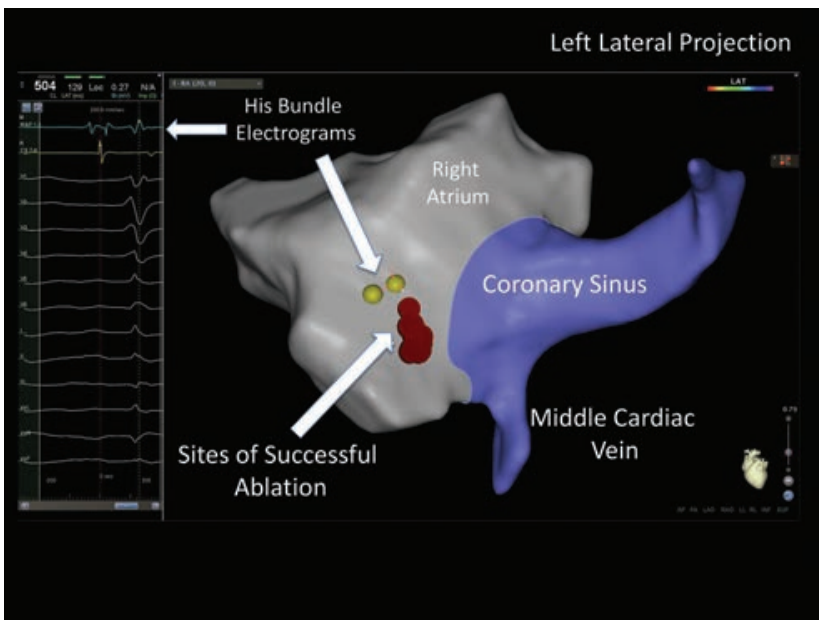

Figure 1: Three-dimensional electroanatomic map of the posteroseptal space generated with the Carto $3^{\text {TM }}$ RMT system (Biosense Webster, Inc, South Diamond Bar, CA). Note the location of the His bundle (yellow spheres) compared with the location of the coronary sinus ostium. Ablation lesions are depicted in red.

pacing cycle lengths. No ventricular tachycardia was inducible. With isoproterenol challenge, supraventricular tachycardia (SVT) consistent with typical AVNRT was induced at a cycle length of $383 \mathrm{~ms}$. Catheter ablation was performed using the Carto $3^{\mathrm{TM}}$ mapping system (Biosense Webster, Inc, South Diamond Bar, CA) in conjunction with Stereotaxis RMN. A 3.5-mm open irrigation RMN catheter was used to perform radiofrequency (RF) ablation of slow pathway potentials with no junctional beats noted during RF energy delivery. It was noted that the patient's His bundle recordings and compact atrioventricular (AV) node were inferiorly displaced from their typical location (Figure 1). This unusual location of these structures was attributed to his prior surgical closured of a perimembranous VSD in early childhood. In 12 months of follow up, the patient has experienced no recurrent tachycardic palpitations or syncopal symptoms.

\section{Discussion}

VSDs constitute approximately $20-30 \%$ of all congenital heart defects, making them the most common type of congenital heart defect. ${ }^{1}$ The ventricular septum is a complex structure composed of three main components: an inlet septum, an apical trabecular septum, and an outlet or infundibular septum. A very small portion of the ventricular septum forms the base of the fibrous triangle between the right and non-coronary leaflets of the aortic valve and is known as the membranous septum. ${ }^{2}$ It consists of both atrioventricular and interventricular components. ${ }^{3}$ The size of this portion of the septum can vary between individuals, and any defect in this area causes elevation of the tricuspid valve annulus to the level of the mitral valve annulus so that all communications are interventricular only. ${ }^{3}$

The ventricular septal complexities become significantly important when considering the various types of VSDs. There are four basic types of VSDs (Table 1): membranous, muscular, inlet, and outlet or supracristal. Membranous VSDs make up the highest percentage, approximately $80 \%$, and can extend into adjacent parts of the septum becoming known as perimembranous defects. ${ }^{1}$ Perimembranous VSDs have part of their border defined by the fibrous continuity between the aortic and tricuspid valves. ${ }^{2}$ Muscular type VSDs are the second most common and make up 5-20\%, whereas less common are outlet-type VSDs $(5-7 \%)$ and isolated inlet VSDs (3-8\%). ${ }^{1}$

The normal conduction system is intricately interwoven into the cardiac structure and can be affected by the presence of VSDs. Normal conduction system anatomy consists of the sinus node, compact AV node, His bundle, and bundle branches. ${ }^{3}$ The AV node is located in the right atrium within the triangle of Koch, which corresponds to the AV septum. ${ }^{3,4}$ The His bundle then arises from the distal portion of the AV node, penetrates through the atrioventricular part of the membranous septum, and ultimately resides along the basal ventricular septum between the membranous and muscular septum. ${ }^{2,3}$

These normal locations for the conduction system become altered in the setting of certain types of VSDs. The AV node and bundle of His are relocated anteriorly and superiorly with an inlet or AV canal type VSD, while they migrate posteriorly and inferiorly in the presence of a membranous VSD. ${ }^{1-3}$ Figure 2 demonstrates the typical locations of the four major types of VSD, including intimate relationship between membranous VSDs and the normal AV nodal conduction system. The anatomic locations of the AV node and His bundle confer significant risk for surgical injury, particularly during repair of membranous VSDs. Following surgical intervention, the anatomic location of these conduction system structures may be altered as further growth and development occurs throughout childhood and into adulthood. ${ }^{5}$

In patients with prior surgical repair of VSDs, special consideration should be given to carefully delineating the location of the normal conduction system during the diagnostic component of electrophysiologic testing prior to ablation delivery. A thorough understanding of the anticipated anatomical changes associated with the different

Table 1: Ventricular septal defect types and relationship to conduction tissue.

\begin{tabular}{lcl}
\hline Type of VSD & Frequency & \multicolumn{1}{c}{ Relationship to conduction tissue } \\
\hline Membranous & $80 \%$ & Atrioventricular node and His bundle located posteriorly and inferiorly to defect \\
Muscular & $5-20 \%$ & Distant from defect \\
Inlet & $3-8 \%$ & Atrioventricular node and His bundle located anteriorly and superiorly to defect \\
Outlet (supracristal) & $5-7 \%$ & Distant from defect \\
\hline
\end{tabular}




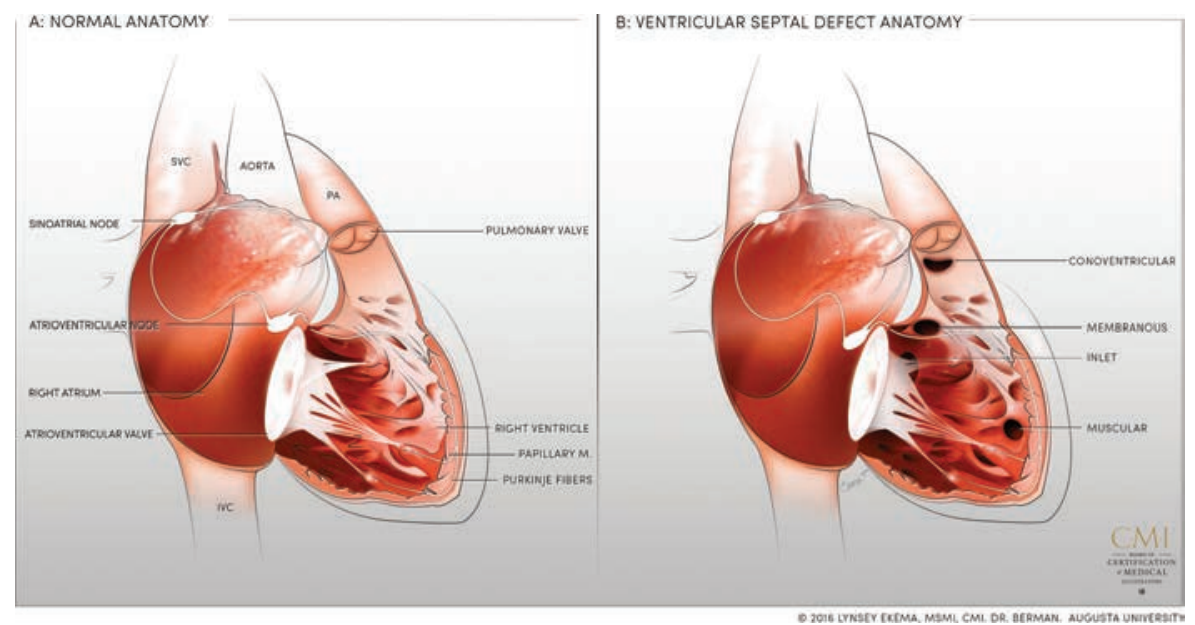

Figure 2: (a) Conduction system location in anatomically normal heart, as viewed from the right atrium and ventricle. (b) Conduction system in relation to ventricular septal defects, as viewed from the right atrium and ventricle. Figure 2 was provided with permission from Lynsey Ekema, MSMI, Medical Illustrator at Augusta University.

forms of VSD is of critical importance when ablating tachycardia originating within or near the compact AV node. In this patient, the post-surgical locations of the compact AV node and His bundle were positioned posteriorly and inferiorly when compared to a structurally normal heart. Indiscriminate slow pathway ablation in territories conventionally regarded as "safe" may have resulted in the creation of iatrogenic complete heart block in this case.

This is the first report in the literature describing the utility of RMN to guide mapping and ablation in the post-surgical VSD population. The precision afforded via RMN-guided catheter ablation was particularly useful in this case as it allowed detailed atraumatic mapping of the slow pathway potentials as well as catheter stability during RF energy delivery. The safety and efficacy of RMN-guided ablation of AVNRT has been demonstrated in non-congenital heart disease patients. Outcomes of RFCA of AVNRT using RMN have been demonstrated to be at least as effective as manual delivery of RF energy. ${ }^{6,7}$ While RMN-guided catheter delivery is not widely available, this case nonetheless highlights the critical importance of a thorough understanding of the original anatomic lesion in patients with underlying congenital heart disease undergoing ablation procedures. If available, a review of surgical records may be especially helpful to the electrophysiologist planning catheter ablation of patients with prior reparative congenital heart surgery. ${ }^{5,8}$

\section{Conclusion}

We describe the initial report of the utility of RMN-guided catheter ablation in a patient with typical AVNRT in the setting of remote surgical repair of a perimembranous VSD. A thorough understanding of the type of VSD that was previously repaired is of critical importance when performing ablation in the region of the $\mathrm{AV}$ node, and should be taken into consideration prior to ablation.

\section{References}

1. Rubio AE, Lewin MB. Ventricular septal defects. In: Allen HD, Driscoll DJ, Shady RE, Feltes TF, eds. Moss and Adams' Heart Disease in Infants, Children, and Adolescents including the Fetus and Young Adult. Philadelphia, PA: Lippincott Williams \& Wilkins; 2013:713-721.

2. McCarthy K, Ho S, Anderson R. Categorisation of ventricular septal defects: Review of the perimembranous morphology. Images Paediatr Cardiol. 2000;2(2):24-40.

3. Edwards WD, Maleszewski JJ. Cardiac anatomy and examination of cardiac Specimens. In: Allen HD, Driscoll DJ, Shaddy RE, Feltes TF, ed. Moss and Adams' Heart Disease in Infants, Children, and Adolescents including the Fetus and Young Adult. Philadelphia, PA: Lippincott Williams \& Wilkins; 2013:1-31.

4. Subramaniam KG, Solomon N. The basis of management of congenital heart disease. In: Firstenberg MS, ed. Principles and Practice of Cardiothoracic Surgery. Rijeka, Croatia: InTech; 2013.

5. Brugada J, Blom N, Sarquella-Brugada G et al. Pharmacological and non-pharmacological therapy for arrhythmias in the pediatric population: EHRA and AEPC-Arrhythmia Working Group joint consensus statement. Europace. 2013; 15(9):1337-1382.

6. Wood MA, Orlov M, Ramaswamy K, Haffajee C, Ellenbogen K; Stereotaxis Heart Study Investigators. Remote magnetic versus manual catheter navigation for ablation of supraventricular tachycardias: A randomized, multicenter trial. Pacing Clin Electrophysiol. 2008;31(10):1313-1321.

7. Shurrab M, Danon A, Crystal A et al. Remote magnetic navigation for catheter ablation of atrioventricular nodal reentrant tachycardia: A systematic review and meta-analysis. Expert Rev Cardiovasc Ther. 2013;11(7):829-836.

8. Kanter RJ. Pearls for ablation in congenital heart disease. J Cardiovasc Electrophysiol. 2010;21(2):223-230. 